\title{
GPURepair: Automated Repair of GPU Kernels
}

\author{
Saurabh Joshi圆 and Gautam Muduganti 圆 * \\ Indian Institute of Technology Hyderabad, India \\ \{sbjoshi,cs17resch01003\}@iith.ac.in \\ VMCAI \\ Artifact \\ Evaluation \\ $\star$ \\ Available

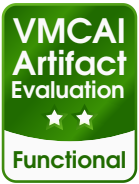 \\ VMCAI \\ Artifact \\ Evaluation \\ $\star \star x+$ \\ Reusable
}

\begin{abstract}
This paper presents a tool for repairing errors in GPU kernels written in CUDA or OpenCL due to data races and barrier divergence. Our novel extension to prior work can also remove barriers that are deemed unnecessary for correctness. We implement these ideas in our tool called GPURepair, which uses GPUVerify as the verification oracle for GPU kernels. We also extend GPUVerify to support CUDA Cooperative Groups, allowing GPURepair to perform inter-block synchronization for CUDA kernels. To the best of our knowledge, GPURepair is the only tool that can propose a fix for intra-block data races and barrier divergence errors for both CUDA and OpenCL kernels and the only tool that fixes inter-block data races for CUDA kernels. We perform extensive experiments on about 750 kernels and provide a comparison with prior work. We demonstrate the superiority of GPURepair through its capability to fix more kernels and its unique ability to remove redundant barriers and handle inter-block data races.
\end{abstract}

Keywords: GPU, Verification, Automated Repair, CUDA, OpenCL

\section{Introduction}

The part of the program that runs on the GPU (Graphics Processing Unit) is referred to as a kernel. Given that multiple cores of the GPU may execute the kernel in parallel, data races and barrier divergence are frequently the cause of several errors that occur in practice. Identifying and repairing these errors early in the development cycle can have a tremendous positive financial impact [8].

In CUDA, a grid consists of blocks, and a block consists of threads. Consider the CUDA kernel in Listing 1.1 without the highlighted line. There is a data race on accesses of the shared array $A$. The race can be avoided by introducing a barrier (_-syncthreads ()) in the kernel at line 3 in Listing 1.1 . This blocklevel barrier enforces that all threads in a block reach it before any of them can proceed further. A grid-level barrier behaves similarly for the entire grid.

\footnotetext{
* The author names are in alphabetical order.
} 
In Listing 1.2 , only the threads with an even thread id will reach the barrier. As the threads within a block execute in a lock-step manner, this will result in a deadlock as threads with odd ids will never be able to reach the barrier at Line 4. This problem is known as barrier divergence.

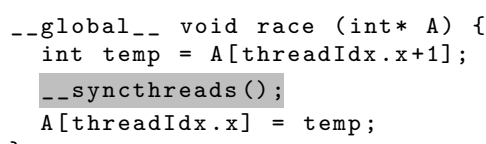

Listing 1.1. Kernel with Data Race

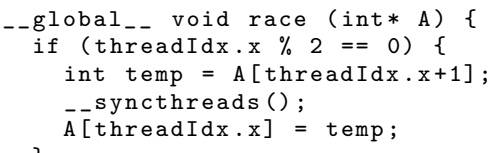

Listing 1.2. Kernel with Barrier Divergence

This tool paper makes the following contributions:

- We extend the underlying technique behind AutoSync [3] to provide barrier placements that avoid barrier divergence in addition to data races. Our novel extension may also suggest removing barriers inserted by the programmer if deemed unnecessary, which might help enhance the performance of the input GPU kernel.

- We implement our technique in our tool GPURepair, which is built on top of the GPUVerify [5] framework and uses GPUVerify as an oracle. To the best of our knowledge, ours is the only technique and tool that can propose a fix for both CUDA and OpenCL GPU kernels. Another unique feature of GPURepair is its ability to fix kernels that have inter-block data races.

- Bugle is the component of the GPUVerify toolchain that translates LLVM bitcode to Boogie. We have enhanced it with the ability to translate barrier synchronization statements from the CUDA Cooperative Groups API to Boogie. We have also extended GPUVerify with the semantics to support grid-level barriers. Using these enhancements, GPURepair proposes fixes for inter-block data races.

- We perform an extensive experimental evaluation on 748 GPU kernels written in CUDA and OpenCL. We compare GPURepair against AutoSync, which is the only other tool known that attempts to repair CUDA kernels containing data races.

\section{GPURepair}

\subsection{GPURepair architecture and workflow}

The implementation of GPURepair builds on top of GPUVerify, as depicted in Fig. 1. In addition to the instrumentation done by GPUVerify to enable verification of GPU kernels, GPURepair adds the instrumentation necessary to impose constraints on the program behavior. On each iteration, GPURepair calls GPUVerify with a proposed solution to check if the program is repaired. If the program is not repaired, it calls the Solver with the constraints generated from the errors seen so far to obtain a candidate solution suggesting which barriers need to be enabled/disabled in the program. If the program can be repaired, GPURepair 


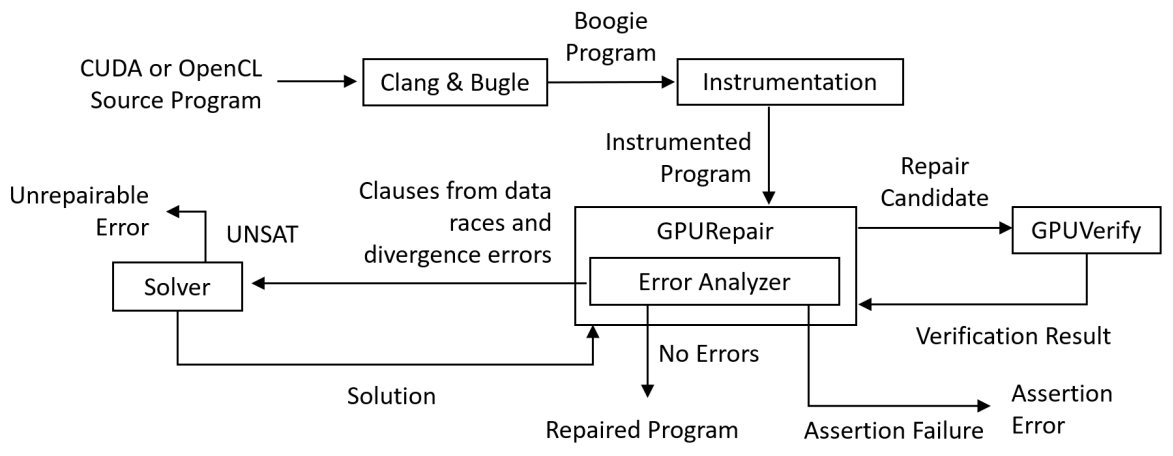

Fig. 1. GPURepair Workflow

generates the Boogie representation of the fixed program and a summary file. The summary file contains the changes that have to be made to fix the program along with the source location details of the original CUDA/OpenCL input kernel. The technique behind GPURepair can, in principle, use any verifier for GPU programs as an oracle.

\subsection{Instrumentation}

GPUVerify uses a pair of distinct non-deterministically chosen threads for analysis instead of modeling all the threads in the kernel. This two-thread abstraction is used to prove that a kernel is race-free and divergence-free. Details of this abstraction are available in [5] and beyond the scope of this paper. GPUVerify models barriers by resetting read/write flags of shared arrays for the two threads used by the two-thread abstraction if they belong to the same block. We extend this to support grid-level barriers for the repair of inter-block data races. A gridlevel barrier is modeled by performing a reset even when the two threads do not belong to the same block.

Since GPURepair attempts to fix errors caused only due to data races or barrier divergence, it proposes a solution that only involves removing existing barriers or adding new ones. Therefore, the instrumentation stage of GPURepair introduces barriers guarded with Boolean variables, referred to as barrier variables. The value of a barrier variable acts as a switch to enable or disable the barrier. A barrier guarded by a barrier variable is referred to as an instrumented barrier. Consider the kernel in Listing 1.3 without the highlighted lines. This kernel has a data race. The instrumentation process adds an instrumented barrier before a shared variable is either read or written. Function calls involving a shared variable are also taken into consideration, and an instrumented barrier is added before the invocation. Pre-existing barriers in the programs are also guarded with barrier variables to explore if they can be removed without introducing data races. 
In addition, the control flow graph (CFG) is analyzed for branch statements and loops to handle scenarios where barriers, if inserted right before the read/write to a shared variable, may introduce barrier divergence. For example, in Listing 1.3 the instrumentation process mentioned above would insert the instrumented barriers right before Line 11 and Line 18 . However, the solution to this program would be a barrier before the if block at Line 15 . The instrumentation process takes these scenarios under consideration by inserting instrumented barriers at the scope boundaries such as entry points of branch statements, loopheads, and function calls. After instrumentation, the highlighted lines at Lines 9 , 13 and 16 in Listing 1.3 are added. For CUDA kernels, if instrumentation of grid-level barriers is enabled, the highlighted lines at Lines 10, 14, and 17 are also added.

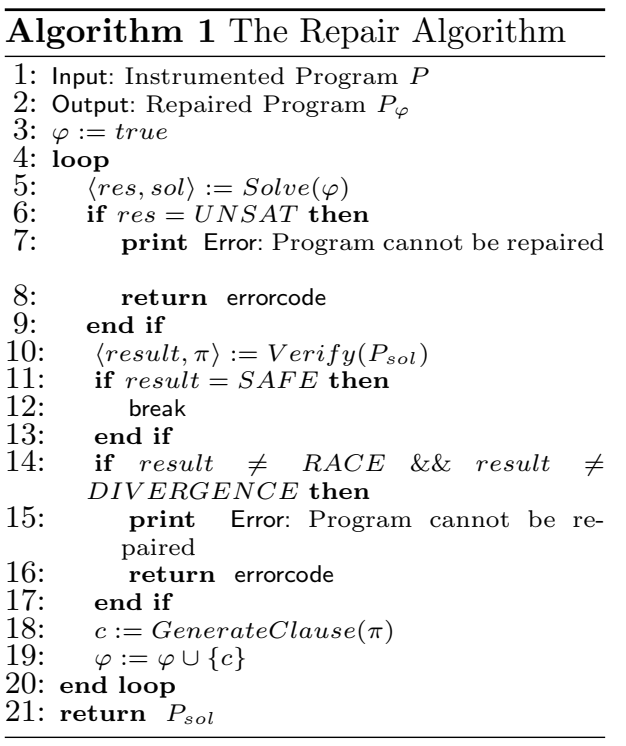

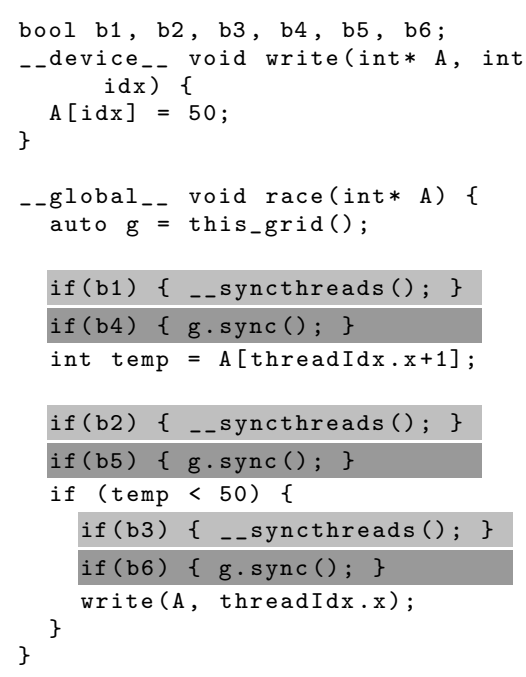

Listing 1.3. Example CUDA Kernel

In this example, variables $\mathrm{b} 1, \ldots, \mathrm{b} 6$ are initially unconstrained. Their values are constrained by GPURepair iteratively to avoid data races or barrier divergence during the repair process. The repair process also assigns weights to these barrier variables such that introducing barriers at the block-level is preferred over the grid-level. This is done because grid-level barriers have a higher performance penalty [32]. For the same reason, barriers nested within loops are less preferred. Although the examples in this section are in CUDA, it should be noted that the actual working of this stage happens on the Boogie program generated by Bugle to make GPURepair agnostic to the front-end language (i.e., CUDA or OpenCL).

\subsection{Preliminaries}

Let $P$ be the given input GPU kernel after instrumentation, as described in Section 2.2. Let $\left\{b_{1}, \ldots, b_{m}\right\}$ be the barrier variables introduced as a part of 
the instrumentation process. Given a formula $\varphi$ over $b_{i}$ 's, let $P_{\varphi}$ denote the instrumented kernel $P$ with values of $b_{i}$ 's constrained to obey $\varphi$.

A clause $c$ is called a positive (resp. negative) monotone clause if it has literals with only positive (resp. negative) polarity (e.g., $b_{1} \vee b_{5} \vee b_{11}$ ). From now on, we may also denote a clause as a set of literals with disjunctions among the set elements being implicit. A formula or a constraint $\varphi$ is a set of clauses with conjunction being implicit among the set elements. Note that a formula $\varphi$ consisting of only positive monotone clauses or only negative monotone clauses is always satisfiable. Let $\varphi^{+}$(resp. $\varphi^{-}$) denote the set of positive (resp. negative) monotone clauses belonging to $\varphi$.

Let $C$ be a set consisting of non-empty sets $S_{1}, \ldots, S_{n}$. The set $\mathcal{H}$ is called a hitting-set (HS) of $C$ if:

$$
\forall_{S_{i} \in C} \mathcal{H} \cap S_{i} \neq \emptyset
$$

$\mathcal{H}$ is called a minimal-hitting-set (mhs) if any proper subset of $\mathcal{H}$ is not a hitting-set. $\mathcal{H}$ is called a Minimum-Hitting-Set $(M H S)$ of $C$ if no smaller hitting set exists for $C$. Note that a collection $C$ may have multiple $m h s$ and multiple $M H S$.

Since we also consider a formula $\varphi$ as a set of clauses, we shall abuse the notation and use $m h s\left(\varphi^{+}\right)$to denote the set of literals that constitutes the minimal-hitting-set of $\varphi^{+}$.

Maximum satisfiability (MaxSAT) is an optimization version of the $S A T$ problem where the goal is to find an assignment that maximizes the number of clauses that are satisfied in a formula. In partial MaxSAT (PMS), given a set of hard clauses $\left(\varphi_{h}\right)$ and a set of soft clauses $\left(\varphi_{s}\right)$, the goal is to find an assignment that satisfies all the clauses of $\varphi_{h}$ while maximizing the number of soft clauses being satisfied. The weighted partial MaxSAT (WPMS) problem asks to satisfy all the hard clauses while maximizing the sum of the weights of the satisfied soft clauses. In WPMS, positive weights are associated with each soft clause.

\subsection{The Repair Algorithm}

Alg. 1 depicts the repair technique behind GPURepair at a high level. It is very similar to the algorithm presented in AutoSync [3].

Initially, all the barrier variables are unconstrained (Line 3), giving the verifier the freedom to set them to any value that leads to an error. Then, Alg. 1 iteratively calls the verifier (Line 4420 until it either finds a solution or determines that it cannot repair the program. A call to the verifier (Line 10 returns an error trace $\pi$ along with the type of the error being captured in result. If the verifier could not find an error (Line 11) with the proposed solution sol, then the algorithm exits the loop, and the instrumented Boogie program constrained with sol is returned (Line 21). If the verifier returned with an error that is neither a data race nor a barrier divergence (Line 14), then Alg. 1 terminates with an error stating that it cannot repair the program. Here, we are operating under the assumption that inserting an extraneous barrier may only introduce a barrier 
divergence error, and removing a programmer-inserted barrier may only cause a data race error.

If the verifier returns with a data race error, then the error trace $\pi$ would tell us which set of barriers were disabled (i.e., the corresponding barrier variables were set to false by the verifier). Let the set of barrier variables that were set to false in $\pi$ be $b_{i_{1}}, \ldots, b_{i_{d}}$. To avoid the same error trace $\pi$ we need to add a constraint represented as a clause $c,\left(b_{i_{1}} \vee, \ldots, \vee b_{i_{d}}\right)$, which is generated by GenerateClause $(\pi)$ (Line 18). Note that such a clause generated from a data race (respectively barrier divergence) error always has only positive (resp. negative) literals. This newly generated clause is added to the constraint $\varphi$ (Line 19, which consists of one clause per error trace. We need to check if $\varphi$ is satisfiable (Line 5). If it is not satisfiable (Line 6), it indicates that there is no assignment to barrier variables that avoids all previously seen traces. Alg. 1 quits with an error (Line 7) in this case. If $\varphi$ is satisfiable, then the Solve method proposes a solution sol (Line 5), which is essentially an assignment to some of the barrier variables. We use two different ways to compute sol from $\varphi$. The first method (the $\operatorname{Max} S A T$ strategy) uses a $\operatorname{Max} S A T$ solver to minimize the number of barrier variables being set to true in sol at each iteration. This is done by solving a partial $\operatorname{Max} S A T$ problem with $\varphi$ as hard clauses and $\left\{\neg b_{1}, \ldots, \neg b_{m}\right\}$ as soft clauses. The second method (the mhs strategy) is to compute a minimalhitting-set $(m h s)$ over $\varphi^{+}$using a polynomial-time greedy algorithm [19] at each iteration to attempt to minimize the number of $b_{i}$ 's being set to true. In this strategy, a single query to a $\operatorname{Max} S A T$ solver is needed to ensure that the number of $b_{i}$ 's being set to true is the minimum.

A similar approach has been used previously in other works [21120]. It should be noted that the clauses generated in these works are all positive monotone clauses (clauses with only positive literals). In contrast, the clauses generated by Alg. 1 could be a mix of positive monotone clauses and negative monotone clauses. Because of this added complication, the approach of using the $m h s$ is not complete. There could be a scenario where the mhs could come up with a solution that causes the negative monotone clauses to be unsatisfiable. Consider an example with the following clauses: $\{b 1 \vee b 3, b 1 \vee b 4, b 2 \vee b 5$, $b 2 \vee b 6, \neg b 1 \vee \neg b 2\}$. The $m h s\left(\varphi^{+}\right)$would give us $b 1$ and $b 2$, which would cause the clause $\neg b 1 \vee \neg b 2$ to be unsatisfiable. To overcome this, GPURepair falls back to the MaxSAT solver whenever the $m h s$ approach results in unsatisfiability.

A barrier inside a loop can pose a heavier performance penalty as opposed to a barrier that is not nested inside a loop. Similarly, inter-block synchronization is more expensive compared to intra-block synchronization 32. In principle, different barrier variables can be given different weights based on loop nesting or profiling information. Then, instead of minimizing the number of barriers, one may want to have barrier placements that minimize the sum of the weights of the enabled barriers. This can easily be achieved by posing this as a weighted mhs or a weighted partial MaxSAT (WPMS) problem. The weight of a barrier is computed as $\left((g w * g b)+l w^{l d}\right)$ where $g w$ is the penalty for a grid-level barrier, 
$g b$ is 0 for block-level barriers and 1 for grid-level barriers, $l w$ is the penalty for a barrier that is inside a loop, and $l d$ is the loop-nesting depth of the barrier.

\section{Related Work}

The verification of GPU programs has been an active area of research for quite a while. GPUVerify [5]4 defines an operational semantics for GPU kernels called synchronous, delayed visibility (SDV) semantics, which mimics the execution of a kernel by multiple groups of threads and uses this to identify data races and barrier divergence. ESBMC-GPU [26] extends the ESBMC model checker by generating an abstract representation of the CUDA libraries and verifies kernels by checking specific desired properties. VerCors 772 builds on permission-based separation logic and extends it to verify GPU programs. PUG [22] is a symbolic verifier that uses SMT solvers to identify bugs in CUDA kernels. Contrary to most of the other verifiers which use static analysis for verification, GKLEE 23] uses concolic verification to identify concrete witnesses for reported bugs.

Automatic program repair is another active area of research that ties-in quite closely with our work. There have been several research efforts in the past for repairing sequential programs [10/16/18/24 as well as concurrent programs $111|17| 28|9| 30|20| 21$. The work done in [11|30|20|21] is very similar to the approach that we take in this paper, where the source code is instrumented, and the repair technique uses the error traces obtained from a verifier to fix the program.

To the best of our knowledge, apart from GPURepair, AutoSync [3] is the only tool that tries to repair a GPU program. AutoSync takes a CUDA program without any barriers and introduces barriers at appropriate locations to remove data race errors.

\subsection{Comparison with AutoSync}

Repair on Source Code vs. Repair on Boogie Code: AutoSync and GPURepair use different types of inputs. AutoSync uses a CUDA program as its input for the repair process, whereas GPURepair uses the Boogie program generated from Bugle. GPURepair is agnostic to the front-end language, allowing it to handle both CUDA and OpenCL. AutoSync, on the other hand, directly takes and manipulates the source code, which makes it fragile to syntactic changes and limits its capabilities to CUDA only.

Consider the kernel in Listing 1.3 . When function inlining is enabled, GPUVerify can accurately identify that Line 3 and Line 11 cause a read-write race through the function call at Line 18 , It reports the line information by specifying that the lines inside the functions cause the read-write race and also explicitly specifies the lines from where these functions are invoked. AutoSync, however, does not process this information correctly and ends up with a code error. GPURepair takes these cases into account and has the ability to place the barrier precisely between the function invocations. 
Barrier Placement vs. Instrumentation: AutoSync uses GPUVerify to identify the lines of code that cause the data race and tries to insert a barrier between these lines of code. In contrast, GPURepair inserts instrumented barriers at various locations in the intermediate Boogie code based on the usage of global variables and uses the trace information provided in errors to enable/disable barriers. This instrumentation gives GPURepair the ability to remove programmerinserted barriers that are deemed unnecessary as well as repair errors caused by barrier divergence or data races that require a grid-level barrier. This feature is exclusive to GPURepair.

Consider the statement $\mathrm{A}[\mathrm{idx}]=\mathrm{A}[\mathrm{idx}+1]$, that has a read-write race occurring in a single line of code. Because AutoSync uses the line information of the statements to identify a read/write on shared variables to insert barriers in the middle, it is unable to do so here since the line numbers will be the same. AutoSync ends up in an infinite loop in these scenarios. In contrast, GPURepair can identify such scenarios since this statement from the source file will be split into two statements, a read followed by a write, in the Boogie representation of the kernel.

Error Parsing vs. SMT Variable Analysis: AutoSync uses regular expressions for parsing the error messages generated by GPUVerify to identify the locations responsible for causing the data race. This technique makes AutoSync extremely fragile to any changes in the output of GPUVerify. For example, AutoSync reports that the program has no errors if, in the output, it does not find texts related to data race or barrier divergence errors. This causes it to misclassify programs with assertion violation errors as error-free programs. GPURepair relies on the SMT model provided by GPUVerify to determine which barriers contributed to the error. This approach makes GPURepair robust and indifferent to the textual output format of GPUVerify.

Inter-block races: GPURepair can propose a fix for inter-block races using CUDA Cooperative Groups for CUDA kernels. No other tool is known to repair inter-block races.

\section{Experiments}

In this section, we present our comparison of GPURepair and AutoSync on several CUDA benchmarks. In addition, we present our findings for runs of GPURepair on OpenCL benchmarks as AutoSync cannot tackle OpenCL kernels. The source code of GPURepair is available at [12. The artifacts and the virtual machine used to reproduce the results of this paper are available at [13] and [31, respectively.

\subsection{Experimental Setup}

Since GPURepair depends on GPUVerify as an oracle, the implementation of GPURepair uses the same technology stack as GPUVerify. The instrumentation and repair stages are built using the .NET Framework with C\# as the programming language. As mentioned in Section 2.1. there are several tools involved in 
the pipeline of GPURepair. Specified below are the version numbers of the tools used in the experimentation. We use the Z3 solver [276] for determining the barrier assignments. The tools used in GPURepair with their versions are: LLVM 6.0.1, Clang 6.0.1, libclc (Revision 353912), Bugle (Revision 15df0ed), GPUVerify (Revision d5a90ec), and Z3 4.6.0.

The experiments were performed on Standard_F2s_v2 Azure ${ }^{\circledR}$ virtual machine, which has 2 vCPUs and $4 \mathrm{GiB}$ of memory. More details on the virtual machine can be found at 25]. For the experiments, a total of 748 kernels (266 CUDA and 482 OpenCL) were considered. This benchmark set is a union of four independent test suites and publicly available [29] sample programs. Tab. 1 summarizes the various sources of the kernels. The average number of lines of code for this benchmark set is 17.51, and the median is 11. 14 kernels have more than 100 lines of code, and 47 have more than 50 lines of code.

Table 1. Benchmark Summary

\begin{tabular}{|l|r|}
\hline \multicolumn{1}{|c|}{ Source } & Kernels \\
\hline \hline GPUVerify Test Suite (Inc. 482 OpenCL Kernels) [15] & 658 \\
\hline NVIDIA GPU Computing SDK v5.0 [29] & 56 \\
\hline AutoSync Micro Benchmarks [3] & 8 \\
\hline $\begin{array}{l}\text { GPURepair Test Suite (Inc. 16 examples for } \\
\text { CUDA Cooperative Groups) }\end{array}$ & 26 \\
\hline
\end{tabular}

All the experiments were performed with a timeout of 300 seconds for each benchmark for each tool. By default, the weight of a grid-level barrier $(g w)$ is taken as 12 , and the weight of a barrier inside a loop $(l w)$ is 10 . For nested loops, the loop-depth is computed, and the loop weight $(l w)$ is raised to the power of the loop-depth.

\subsection{Results}

Tab. 2 summarizes the results obtained from running the benchmark suite with GPURepair and AutoSync. AutoSync does not support OpenCL; therefore, results for OpenCL are only applicable for GPURepair. Numbers in bold indicate better results.

The table categorizes the results into three categories based on the output of GPUVerify. The first category includes all the kernels for which GPUVerify concluded that there were no errors. For all 152 CUDA kernels that fall in this category, AutoSync crashed in 6 of these benchmarks, and even though GPUVerify did not give any errors, GPURepair suggested removal of unnecessary barriers for 13 CUDA kernels and 25 OpenCL kernels. Removal of unnecessary barriers is a feature exclusive to GPURepair. AutoSync attempts only to insert barriers so as to avoid data race errors. 
Table 2. Count of kernels grouped by category

\begin{tabular}{|l|r|r|r|}
\hline \multicolumn{1}{|c|}{ Category } & AutoSync & \multicolumn{2}{|c|}{ GPURepair } \\
\hline Total Benchmarks & CUDA & CUDA & OpenCL \\
& 266 & 266 & 482 \\
\hline \hline Verified by GPUVerify & 152 & 152 & 331 \\
\hline No changes made by the tool & 146 & 138 & 293 \\
Changes recommended by the tool & 0 & $\mathbf{1 3}$ & 25 \\
Errors & 6 & $\mathbf{0}$ & 10 \\
Timeouts (300 seconds) & 0 & 1 & 3 \\
\hline \hline Data Race/Barrier Divergence Errors & 89 & 89 & 69 \\
identified by GPUVerify & 31 & $\mathbf{4 3}$ & 33 \\
\hline Repaired by the tool & 0 & $\mathbf{1 5}$ & 0 \\
Repaired using grid-level barriers & 10 & 20 & 34 \\
Could not be repaired by the tool & 14 & $\mathbf{0}$ & 0 \\
Errors & 34 & $\mathbf{1 1}$ & 2 \\
Timeouts (300 seconds) & 25 & 25 & 82 \\
\hline \hline Unrepairable errors identified by & 0 & $\mathbf{2 5}$ & 82 \\
GPUVerify & 24 & $\mathbf{0}$ & 0 \\
\hline Handled gracefully by the tool & 1 & $\mathbf{0}$ & 0 \\
False Positives & & \\
Errors & & & \\
\hline
\end{tabular}

The second category includes the kernels for which GPUVerify had identified data races or barrier divergence errors. For 10 benchmarks, AutoSync stated that the error could not be repaired. Out of these 10, GPURepair was able to repair 6, it timed out for 2, and it could not repair 2 of these. The final category involves the kernels that had either assertion errors or errors thrown by Clang or Bugle or had invalid invariants. Repairing these kernels is beyond the scope of either AutoSync or GPURepair. AutoSync claimed that a solution was found for 24 out of the 25 CUDA kernels in this category, but those were found to be false positives. AutoSync checks the textual error messages for any information related to data races and barrier divergence, and if it does not find anything, AutoSync treats it as a success. In contrast, GPURepair returned the same error code as GPUVerify for all the benchmarks in this category. This category highlights the fragile nature of AutoSync, as it syntactically depends on the output of GPUVerify.

It is evident that GPURepair provides much more coverage as it can handle OpenCL kernels. Even for CUDA kernels, GPURepair provides better coverage as it can repair more programs, support inter-block synchronization using CUDA Cooperative Groups, and exits gracefully for a larger number of kernels.

As described in Tab. 2, there were 25 benchmarks in the third category where GPUVerify itself throws an irreparable error (e.g., either an assertion violation or errors thrown by other stages). We provide a time comparison in the form of a scatter plot shown in Fig. 2 for the remaining 241 CUDA benchmarks, which are 


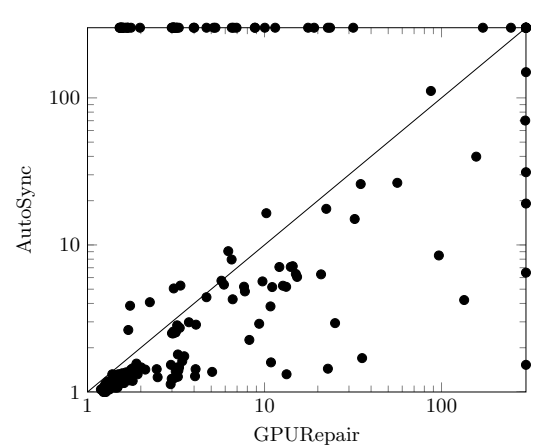

Fig. 2. Runtime in seconds (Log Scale)

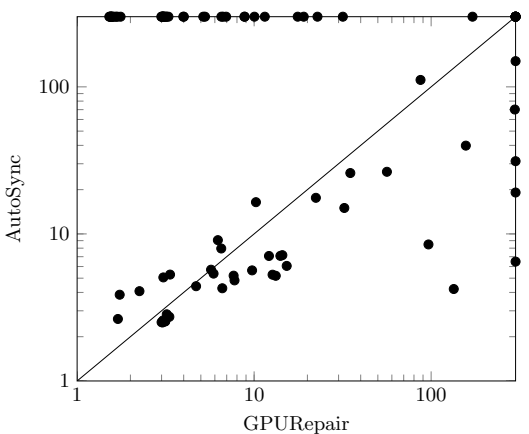

Fig. 3. Runtime in seconds - Repair Candidates (Log Scale)

either certified as error-free by GPUVerify or contain data race and/or barrier divergence errors. Each benchmark has been executed 3 times for each of the tools, and the average time for these 3 runs is taken into consideration. We used the average since there was a negligible difference between the median and the average.

Out of the 241 benchmarks, AutoSync was faster in 178 cases, whereas GPURepair was faster for 63 benchmarks. GPUVerify did not show any error for 152 out of these 241 benchmarks. For these benchmarks, AutoSync did not have to put any further efforts. In contrast, GPURepair attempts to determine if there are any programmer-inserted barriers that are unnecessary and could be removed. This explains GPURepair being slower for some of the benchmarks. Fig. 3 shows a run time comparison for 89 benchmarks for which GPUVerify found data race/barrier divergence errors. GPURepair performs significantly better than AutoSync when a kernel requires repair. This is evident from Fig. 3 as AutoSync was faster on 32 benchmarks, whereas GPURepair was faster on 57 benchmarks out of these 89 benchmarks. Note that if any of the tools crash on a benchmark, we consider that run to have timed out; that is, as a benchmark run that took 300 seconds.

The default configuration of GPURepair uses the mhs strategy to solve the clauses, enables exploring grid-level barriers to find a solution, and inspects pre-existing barriers for removal if deemed unnecessary. The solver type can be changed to MaxSAT, and the usage of grid-level barriers and inspection of preexisting barriers can be disabled through command-line options. In addition, the weight of grid-level barriers and the weight of barriers nested within loops can also be overridden using command-line options. A.5 gives a brief description on how to run GPURepair; detailed documentation can be found at [12]. Tab. 3 compares the time taken by AutoSync and GPURepair on different configurations. The total time taken by AutoSync is higher, primarily because 300 seconds were counted every time any of the tools crashed. This analysis demonstrates that GPURepair is more robust than AutoSync while performing quite close to AutoSync with respect to runtime. 
Table 3. Comparison of AutoSync and the various configurations of GPURepair

\begin{tabular}{|l|r|r|r|r|r|}
\hline & \multicolumn{2}{|c|}{$\begin{array}{c}\text { All Kernels } \\
(241)\end{array}$} & \multicolumn{3}{c|}{$\begin{array}{c}\text { Repaired + Unchanged } \\
(28+133=161)\end{array}$} \\
\hline $\begin{array}{c}\text { Tool } \\
\text { (Configuration) }\end{array}$ & $\begin{array}{c}\text { Total } \\
\text { Time }\end{array}$ & $\begin{array}{c}\text { Median } \\
\text { Time } \\
\text { (in seconds) }\end{array}$ & $\begin{array}{c}\text { Total } \\
\text { Time }\end{array}$ & $\begin{array}{c}\text { Median } \\
\text { Time }\end{array}$ & $\begin{array}{c}\text { Verifier } \\
\text { Calls }\end{array}$ \\
\hline \hline AutoSync & 17076 & 1.43 & 384 & 1.24 & 216 \\
\hline GPURepair & 5810 & 1.76 & 823 & 1.57 & 271 \\
\hline GPURepair --maxsat & 5940 & 1.75 & 887 & 1.54 & 306 \\
\hline GPURepair --disable-grid & 4450 & 1.72 & 660 & 1.56 & 254 \\
\hline $\begin{array}{c}\text { GPURepair } \\
\text {--disable-inspect }\end{array}$ & 5430 & 1.81 & 754 & 1.56 & 250 \\
\hline $\begin{array}{c}\text { GPURepair --disable-grid } \\
\text {--disable-inspect }\end{array}$ & 4225 & 1.79 & 621 & 1.51 & 235 \\
\hline
\end{tabular}

Additional experiments and analysis are included in Appendix A which provides more experimental details of the various configurations of GPURepair (A.1), the code size of the benchmarks A.2, analysis of the errors encountered by AutoSync A.3), case studies A.4, and how to run GPURepair A.5.

\section{Conclusion}

This tool paper introduces GPURepair, which can fix barrier divergence errors and remove unnecessary barriers in addition to AutoSync's ability to fix data races. GPURepair has the additional capability to handle both CUDA and OpenCL kernels. Most importantly, GPURepair has a unique feature for suggesting a fix for inter-block races using Cooperative Groups in CUDA.

With extensive experimental evaluation on the benchmark suites (consisting of 700+ CUDA/OpenCL kernels), we have affirmed the superiority of our work. Our experimental results clearly show that GPURepair provides far more coverage than AutoSync. For $65 \%$ of the total benchmarks, AutoSync was not applicable as 482 out of the 748 benchmarks were OpenCL kernels. Even for CUDA kernels, GPURepair is able to provide more coverage and is able to repair more kernels. GPURepair is also faster than AutoSync when a kernel indeed requires repair.

\section{Acknowledgements}

We thank the anonymous reviewers for their helpful comments and the authors of AutoSync for providing the source-code under a public license. We also thank the Ministry of Education, India, for financial support. 


\section{References}

1. Abdulla, P.A., Atig, M.F., Chen, Y., Leonardsson, C., Rezine, A.: Counter-example guided fence insertion under TSO. In: TACAS 2012. pp. 204-219. Springer (2012)

2. Amighi, A., Darabi, S., Blom, S., Huisman, M.: Specification and verification of atomic operations in GPGPU programs. In: SEFM 2015. pp. 69-83. Springer (2015)

3. Anand, S., Polikarpova, N.: Automatic synchronization for GPU kernels. In: FMCAD 2018. pp. 1-9. IEEE (2018)

4. Betts, A., Chong, N., Donaldson, A.F., Ketema, J., Qadeer, S., Thomson, P., Wickerson, J.: The design and implementation of a verification technique for GPU kernels. TOPLAS 37(3), 10:1-10:49 (2015)

5. Betts, A., Chong, N., Donaldson, A.F., Qadeer, S., Thomson, P.: GPUVerify: a verifier for GPU kernels. In: OOPSLA 2012. pp. 113-132. ACM (2012)

6. Bjørner, N., Phan, A., Fleckenstein, L.: $\nu$ z - an optimizing SMT solver. In: TACAS 2015. pp. 194-199. Springer (2015)

7. Blom, S., Huisman, M., Mihelcic, M.: Specification and verification of GPGPU programs. Science of Computer Programming 95, 376-388 (2014)

8. Boehm, B.W., Papaccio, P.N.: Understanding and controlling software costs. IEEE Trans. Software Eng. 14(10), 1462-1477 (1988)

9. Cerný, P., Chatterjee, K., Henzinger, T.A., Radhakrishna, A., Singh, R.: Quantitative synthesis for concurrent programs. In: CAV 2011. pp. 243-259. Springer (2011)

10. Chandra, S., Torlak, E., Barman, S., Bodík, R.: Angelic debugging. In: ICSE 2011. pp. 121-130. ACM (2011)

11. Deshmukh, J.V., Ramalingam, G., Ranganath, V.P., Vaswani, K.: Logical concurrency control from sequential proofs. In: ESOP 2010. pp. 226-245. Springer (2010)

12. GPURepair: GPURepair GitHub Repository (https://github.com/cs17resch01003/gpurepair). GPURepair (2020)

13. GPURepair: GPURepair VMCAI 2021 Artifacts (https://doi.org/10.5281/zenodo.4276525). GPURepair (2020)

14. GPUVerify: GPUVerify Command-Line Options (https://github.com/mcimperial/gpuverify/blob/master/Documentation/basic_usage.rst). GPUVerify (2020)

15. GPUVerify: GPUVerify Test Suite (https://github.com/mcimperial/gpuverify/tree/master/testsuite). GPUVerify (2020)

16. Griesmayer, A., Bloem, R., Cook, B.: Repair of boolean programs with an application to C. In: CAV 2006. pp. 358-371. Springer (2006)

17. Jin, G., Song, L., Zhang, W., Lu, S., Liblit, B.: Automated atomicity-violation fixing. In: PLDI 2011. pp. 389-400. ACM (2011)

18. Jobstmann, B., Griesmayer, A., Bloem, R.: Program repair as a game. In: CAV 2005. pp. 226-238. Springer (2005)

19. Johnson, D.S.: Approximation algorithms for combinatorial problems. Journal of Computer and System Sciences 9(3), 256-278 (1974)

20. Joshi, S., Kroening, D.: Property-driven fence insertion using reorder bounded model checking. In: FM 2015. pp. 291-307. Springer (2015)

21. Joshi, S., Lal, A.: Automatically finding atomic regions for fixing bugs in concurrent programs. CoRR abs/1403.1749 (2014)

22. Li, G., Gopalakrishnan, G.: Scalable smt-based verification of GPU kernel functions. In: FSE 2010. pp. 187-196. ACM (2010) 
23. Li, G., Li, P., Sawaya, G., Gopalakrishnan, G., Ghosh, I., Rajan, S.P.: GKLEE: concolic verification and test generation for gpus. In: PPOPP 2012. pp. 215-224. ACM (2012)

24. Malik, M.Z., Siddiqui, J.H., Khurshid, S.: Constraint-based program debugging using data structure repair. In: ICST 2011. pp. 190-199. IEEE Computer Society (2011)

25. Microsoft: Microsoft Azure Fsv2-Series Virtual Machine Sizes (https://docs.microsoft.com/en-us/azure/virtual-machines/fsv2-series). Microsoft Corporation (2020)

26. Monteiro, F.R., da S. Alves, E.H., da Silva, I., Ismail, H., Cordeiro, L.C., de Lima Filho, E.B.: ESBMC-GPU A context-bounded model checking tool to verify CUDA programs. Science of Computer Programming 152, 63-69 (2018)

27. de Moura, L.M., Bjørner, N.: Z3: an efficient SMT solver. In: TACAS 2008. pp. 337-340. Springer (2008)

28. Muzahid, A., Otsuki, N., Torrellas, J.: Atomtracker: A comprehensive approach to atomic region inference and violation detection. In: MICRO 2010. pp. 287-297. IEEE Computer Society (2010)

29. Nvidia: CUDA Toolkit 5.0 (https://developer.nvidia.com/cuda-toolkit-50-archive). Nvidia Corporation (2019)

30. Vechev, M.T., Yahav, E., Yorsh, G.: Abstraction-guided synthesis of synchronization. In: POPL 2010. pp. 327-338. ACM (2010)

31. VMCAI: VMCAI 2021 Virtual Machine (https://doi.org/10.5281/zenodo.4017292). VMCAI (2020)

32. Zhang, L., Wahib, M., Zhang, H., Matsuoka, S.: A study of single and multi-device synchronization methods in nvidia gpus. In: 2020 IEEE International Parallel and Distributed Processing Symposium (IPDPS), New Orleans, LA, USA, May 18-22, 2020. pp. 483-493. IEEE (2020)

\section{Appendix A More Experiments and Results}

\section{A.1 Configuration Comparison}

As mentioned in Section 2.4. GPURepair computes the minimal-hitting-set ( $m h s$ ) of the clauses obtained in each run of the algorithm. Using $m h s$ can avoid expensive calls to the $\operatorname{Max} S A T$ solver at every iteration. In principle, using the mhs strategy should always outperform the $\operatorname{Max} S A T$ strategy if the same set of error traces are seen in the same sequence. However, since the approach with/without $m h s$ would result in different sets of traces being witnessed and in different sequences, mhs may not always outperform on every benchmark. For example, consider that in the first iteration, we obtain a clause $a \vee b$ from GPUVerify. $m h s$ could choose $a$ to be the solution, and MaxSAT could choose $b$ to be the solution. Both of the solutions are valid for the clause, but choosing $b$ could fix the kernel, and choosing a may not, thus forcing more iterations. These "lucky" choices can introduce deviations regarding which solver would perform better on different benchmarks. Fig. 4 shows the runtime comparison of these solvers with a 300 seconds timeout for each benchmark.

The $m h s$ (default) strategy of GPURepair takes lesser time to run all the 748 benchmarks compared to the MaxSAT strategy, taking a total time of 9145 
seconds. When grid-level barriers and inspection of programmer-inserted barriers are disabled, the time reduces to 6467 seconds, but this comes at the cost of not repairing a few kernels that require grid-level synchronization and not optimizing any.

The $m h s$ strategy of GPURepair made a total of 415 calls to Z3. Note that these do not include the calls made to Z3 by GPUVerify. There were 9 kernels for which $m h s$ could not solve the input clauses (a possibility explained in Section 2.4 , and the MaxSAT solver was used as a fallback. In all of these cases, the MaxSAT solver also returned UNSAT.

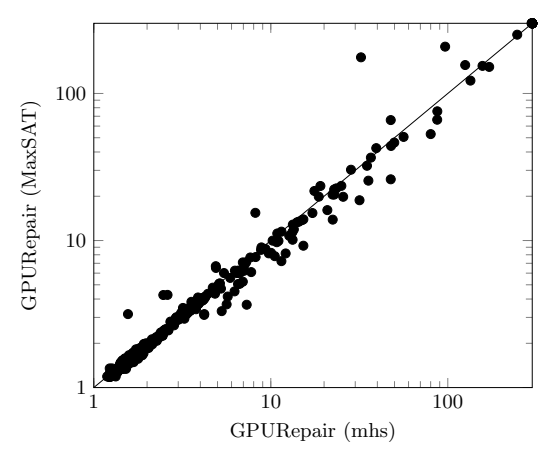

Fig. 4. Runtime in seconds - mhs vs. MaxSAT (Log Scale)

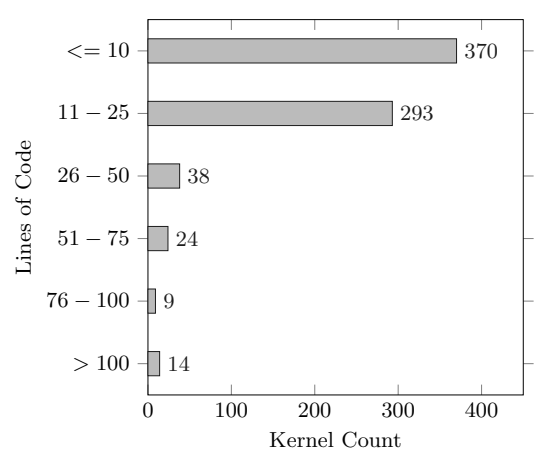

Fig. 5. Lines of Code

\section{A.2 Source Code Size}

Fig. 5 shows the size of the kernels in terms of lines of code. The average number of lines of code for the 748 kernels in the evaluation set was 17.51, and the median was 11.14 kernels had more than 100 lines of code, and 47 had more than 50 lines of code. The majority of the kernels had less than 25 lines of code. The kernel with the highest number of lines of code had 639 lines.

The instrumentation step of GPURepair happens on the Boogie code and not on the source code. A line of source code could result in zero (e.g., code comments) or more Boogie commands. Fig. 6 shows the size of the kernels in terms of Boogie commands. The average number of Boogie commands for the 734 kernels for which Boogie code was generated was 25.72, and the median was 11. The majority of the kernels had less than 25 commands. The kernel with the highest number of Boogie commands had 1793 commands.

The repair step of GPURepair depends on the number of barriers that are instrumented in code, which depend on the number of shared variables and how many times they have been used.

Fig. 7 shows the number of barrier variables introduced in the instrumentation stage of GPURepair. This also includes the barrier variables introduced 


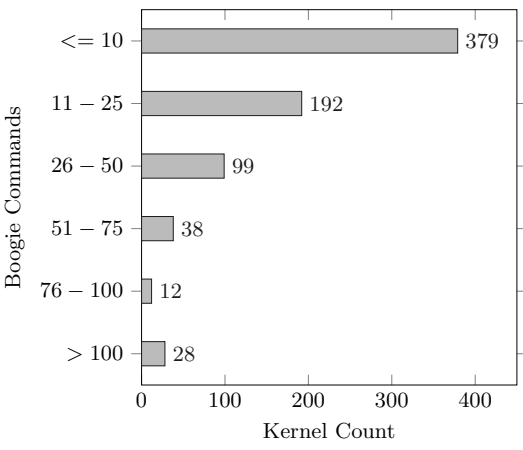

Fig. 6. Boogie Commands

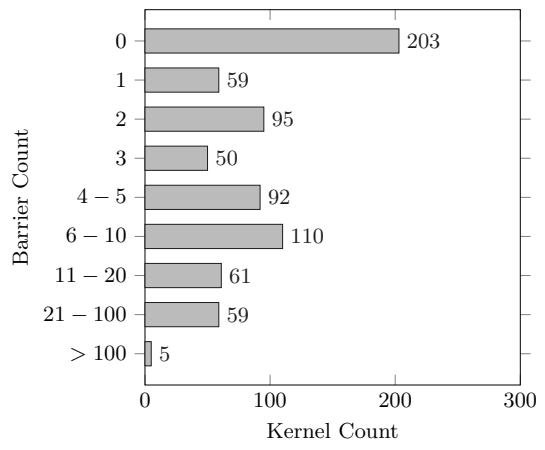

Fig. 7. Kernels with 'n' Instrumented Barriers

for existing barriers provided by the programmer. Out of the 734 kernels that reached the instrumentation stage (inclusive of CUDA and OpenCL kernels), more than $50 \%$ had less than three instrumented barriers introduced during the instrumentation stage, and there were 5 kernels with more than 100 instrumented barriers. The highest number of barriers, 588, were introduced in the OpenCL/ vectortests/float4initialisation test-case, followed by CUDASamples/6_ Advanced_shfl_scan_shfl_intimage_rows, which had 356 instrumented barriers. For all the kernels, the time taken by the instrumentation stage was less than a second.

\section{A.3 AutoSync Errors}

AutoSync crashes for 21 kernels. The root cause of these crashes can be classified into two broad categories - error message parsing and source code parsing.

8 kernels crash because of error message parsing. These kernels have a method named "race" that causes the problem. GPUVerify throws a warning about aliasing, which mentions the method name, and AutoSync mistakes it for an error related to data race because of the regular expressions that are used for error parsing. For 3 kernels, changing the method name worked, and AutoSync was able to repair them. The solution for 3 of these kernels involved inter-block synchronization, which AutoSync does not support. For the remaining 2, AutoSync timed out.

The remaining 13 kernels crash because of source code parsing. AutoSync has a component that initially parses the source code, which is used at a later stage to decide where a barrier can be inserted. 6 kernels crashed because the if-else statements had the opening brace in the same line as the if statement. After moving the brace to a new line, AutoSync was able to work for 5 of the kernels. For the sixth kernel, AutoSync returned a false-positive result since the kernel had a Bugle error. The remaining 7 kernels crashed because of complicated source code parsing issues in AutoSync. We were not able to find a fix in the AutoSync 
code for these. This component ideally would need to be a full-fledged $\mathrm{C}$ parser, which is quite cumbersome to build.

\section{A.4 Case Studies}

In this section, we showcase some of the examples that needed special consideration while designing GPURepair. Consider Listing 1.4 there is a read-write race between Line 5 and Line 6. The repair algorithm identifies this and inserts a barrier before Line 6. However, since these statements are inside an if block, adding the barrier leads to a barrier divergence. The conflict between having the barrier and encountering a divergence error and not having it and encountering a race causes GPURepair to consider it an unrepairable kernel. Neither GPURepair nor AutoSync can repair such kernels.

Listing 1.5 showcases an example of a shared array being read and written to in the same statement. When Bugle transforms this kernel to Boogie, it breaks these statements into two statements, as shown below. GPURepair leverages this to recommend a barrier placement between the read and write to fix the kernel. As mentioned in Section 3.1. AutoSync cannot handle such scenarios.

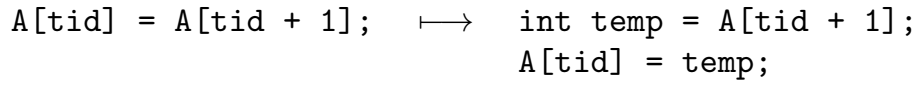

One of the scenarios that we came across in the GPUVerify test suite was a write-write race by multiple threads on the same shared array at the same index. This is shown in Listing 1.6. There is no solution for such kernels, and neither GPURepair nor AutoSync can fix them.

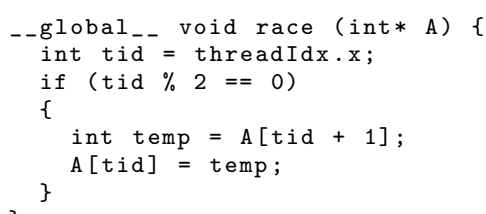

Listing 1.4. Unrepairable Kernel

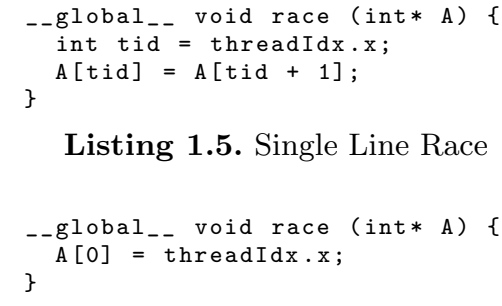

Listing 1.6. Write-Write Race

Consider the kernel in Listing 1.7. When function inlining is enabled, GPUVerify can accurately identify that Line 12 and Line 13 cause a read-write race through the functions invoked at these lines of code. It reports the line information by specifying that the lines inside the functions cause the read-write race and also explicitly specifies the lines from where these functions are invoked. AutoSync, however, does not process this information correctly and ends up with a code error. GPURepair takes these cases into account and can place the barrier precisely between the function invocations. 


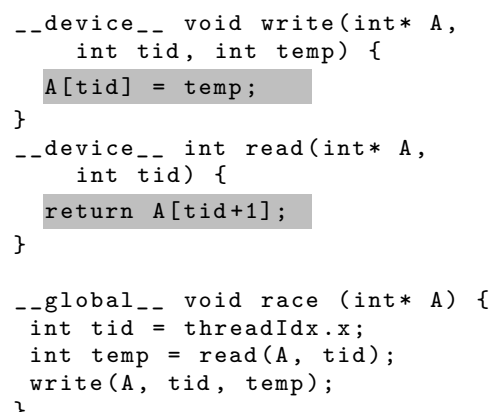

Listing 1.7. Inter Function Race

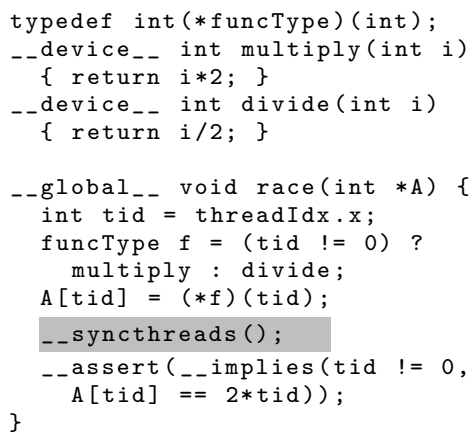

Listing 1.8. Assertion Errors after Adding Barriers

Consider the kernel in Listing 1.8 . Without the barrier at the highlighted line, GPUVerify can successfully verify and conclude that there are no errors. However, when the barrier is introduced at Line 12. GPUVerify reports an assertion error. This false positive is due to the way GPUVerify abstracts shared state when it encounters a barrier in such cases. This happens even when the barrier is an instrumented barrier. Due to scenarios like these, sometimes GPURepair is unable to obtain a solution even though one exists. AutoSync also needs the verifier to be sound and complete for it to find a solution.

\section{A.5 Running GPURepair}

As emphasized in Fig. 1, GPURepair uses multiple tools in its repair process. We provide a python script, which takes care of calling all of the tools in the toolchain in the appropriate order. Once the kernel is repaired, GPURepair prints the needed changes in the source code and provides a fixed Boogie program as evidence. Fig. 8 shows a sample run and the changes recommended by GPURepair for this kernel.

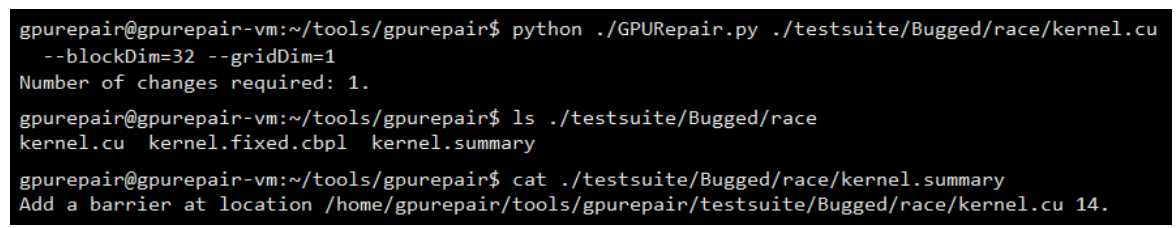

Fig. 8. GPURepair Commands

GPUVerify supports several verification options. These command-line options are documented at 14. All of these can be used with GPURepair as well. GPURepair passes these command-line options, if specified during its invocation, to GPUVerify for the verification process. Along with these, GPURepair also provides 
specific options for the repair process. A description of these can be accessed from the help section. Fig. 9 shows this.

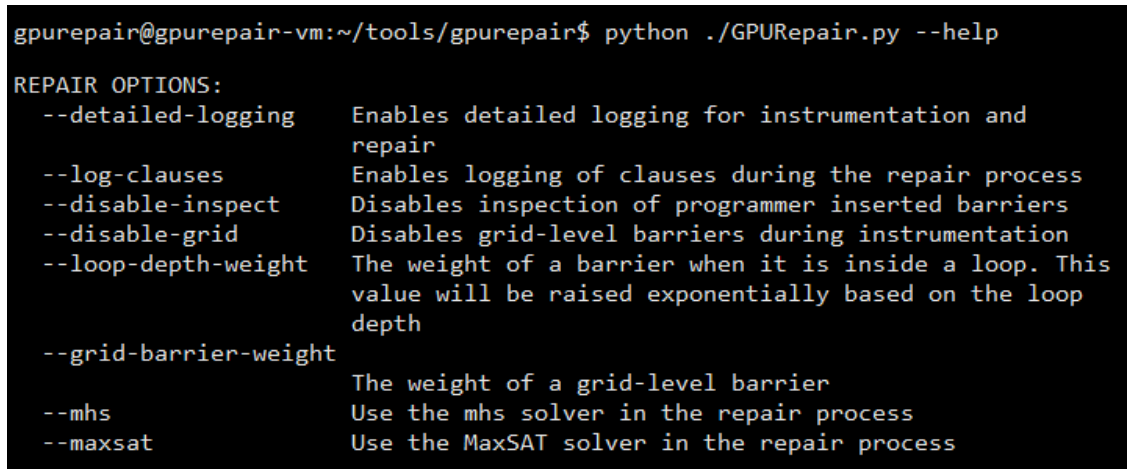

Fig. 9. GPURepair Repair Options 